\title{
Adaptive optics full-field optical coherence tomography
}

Peng Xiao

Mathias Fink

Albert Claude Boccara 


\title{
Adaptive optics full-field optical coherence tomography
}

\author{
Peng Xiao, Mathias Fink, and Albert Claude Boccara* \\ PSL Research University, Institut Langevin, ESPCI Paris, 1 rue Jussieu, 75005 Paris, France
}

\begin{abstract}
We describe a simple and compact full-field optical coherence tomography (FFOCT) setup coupled to a transmissive liquid crystal spatial light modulator (LCSLM) to induce or correct aberrations. To reduce the system complexity, strict pupil conjugation was abandoned because low-order aberrations are often dominant. We experimentally confirmed a recent theoretical and experimental demonstration that the image resolution was almost insensitive to aberrations that mostly induce a reduction of the signal level. As a consequence, an imagebased algorithm was applied for the optimization process by using the FFOCT image intensity as the metric. Aberration corrections were demonstrated with both an USAF resolution target and biological samples for LCSLM-induced and sample-induced wavefront distortions. () 2016 Society of Photo-Optical Instrumentation Engineers (SPIE) [DOI: 10.1117/1.JBO.21.12.121505]
\end{abstract}

Keywords: optical coherence tomography; interference microscopy; adaptive optics.

Paper 160433SSPR received Jun. 23, 2016; accepted for publication Sep. 7, 2016; published online Sep. 22, 2016.

\section{Introduction}

Biological tissues are heterogeneous systems that strongly scatter light. In order to obtain images of in-depth structures hindered by scattering, one must be able to select ballistic or, more precisely, singly backscattered photons. This can be achieved by a number of optical approaches, such as confocal microscopy, ${ }^{1}$ multiphoton microscopy, ${ }^{2}$ or optical coherence tomography ${ }^{3}$ (OCT). The use of OCT has increased dramatically in various research and clinical studies since its development, especially in ophthalmology, due to its noninvasiveness and high imaging speed. Our laboratory has developed a specific "en face" approach of OCT, full-field OCT or FFOCT, that uses incoherent broadband light sources coupled to imaging interferometers (e.g., Linnik) to select optical slices perpendicular to the optical axis. ${ }^{4}$ These systems do not require the usual large depth of field of standard OCT approaches and, thus, allow obtaining micron scale resolution in three-dimensional by the use of microscope objectives.

If small-scale heterogeneities induce scattering, there are also multiscale aberrating structures in the eye or tissues that reduce the high-resolution image quality. Thus, building a wavefront adaptive system is strongly needed to achieve diffraction limited imaging. Adaptive optics (AO) was originally proposed and developed for astronomical imaging of optical telescopes to correct the atmosphere-induced wavefront perturbations. ${ }^{5,6}$ In recent years, $\mathrm{AO}$ has found valuable applications to correct biological tissue-induced aberrations in biological and medical imaging, ${ }^{7}$ especially for retinal imaging in order to visualize cellular structures. ${ }^{8-10} \mathrm{AO}$-assisted fundus photography, ${ }^{11}$ scanning laser ophthalmoscopy, ${ }^{12-14}$ and $\mathrm{OCT}^{15-22}$ systems have achieved reliable images of cones and rods photoreceptors.

In general, the correction in many AO systems such as twophoton microscopy ${ }^{23}$ and AO-OCT systems ${ }^{15,16,18-22,24}$ uses a

*Address all correspondence to: Albert Claude Boccara, E-mail: claude. boccara@espci.fr conjugation of the image focal plane of the microscope objective or of the eye pupil with the wavefront sensors or correction devices. This strict pupil conjugation appears to be mandatory when very high-order aberrations are involved because one cannot rely on simple geometrical optics propagation of the wavefront but one has to account for diffractive effects of wave propagation. However, the telescopic systems needed to achieve strict pupil conjugation increase the system complexity and the optical path length, which has to be balanced within less than $1 \mu \mathrm{m}$ due to the axial sectioning of FFOCT.

As the ultimate goal of our study is to apply FFOCT for human eye examination, the problem appears different since low-order aberrations dominate in the eye. Several studies on eye aberrations have shown that the majority of the Zernike polynomials that are involved in a large number of eyes aberrations tests are mostly low-order ones, ${ }^{25-27}$ meaning that at different steps of the propagation, the wavefront looks like a homothetic image of itself. In order to overcome complex setups realizations and to be able to apply FFOCT to low-order aberration correction, we intend to use transmissive liquid crystal spatial light modulators ${ }^{28-32}$ (LCSLMs) that could be roughly positioned for wavefront distortion correction, analogous to commonly used spectacles for correcting eye's myopia and astigmatism. While many kinds of wavefront correctors have been developed and applied for eye's diffraction limit imaging, they all have pros and cons in parameters, such as temporal bandwidth, reflectivity, mirror diameter, and number of actuators. ${ }^{33}$ LCSLM fits for our application as it can work in a transmissive way with a large number of pixels and a low control voltage. Let us note that LCSLMs have already been used to alter the refractive state ${ }^{31}$ and to correct the aberrations of the eye. ${ }^{32}$ Nevertheless in both cases pupil conjugation using telescopes have been used. As mentioned before we intend to get rid of these telescopes in order to simplify the setup. Of course the confined $2 \pi$ phasemodulation range of LCSLM may limit the correction of 


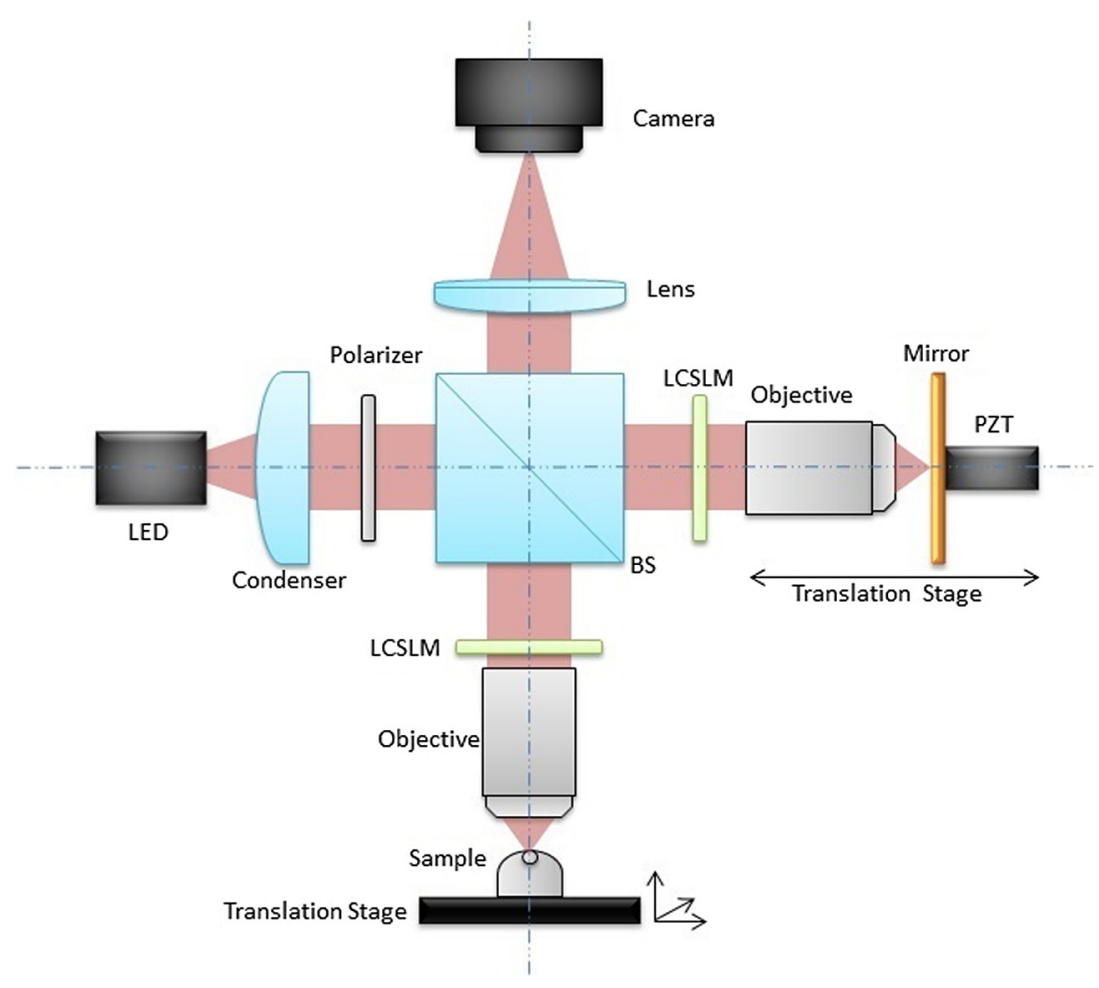

Fig. 1 Schematic of AO FFOCT system coupled with LCSLMs. BS, beamsplitter; LCSLM, liquid crystal spatial light modulator; PZT, piezoelectric transducer.

aberrations with large magnitudes; nevertheless, the adjusting range is doubled as the incoming and outgoing beams both induce optical path difference in our system. Phase wrapping could also be used to extend the dynamic range. ${ }^{31,34}$

Last but not least we would like to avoid measuring the wavefront. In most AO systems, direct wavefront measurements are usually conducted with a wavefront sensor or coherencegated wavefront sensing in a closed-loop configuration together with a wavefront corrector. But due to the lack or generally usable wavefront sensors and the inherent complexity of the coherent wavefront sensing, considerable interests have been focused on wavefront sensorless methods, such as hill climbing, ${ }^{35}$ genetic algorithm, ${ }^{36}$ simulated annealing, ${ }^{37}$ and pupil segmentation. ${ }^{38}$ Recently, we discovered that, unlike scanning OCT in spatially incoherent interferometry like FFOCT, aberrations do not affect the width of the system point spread function (PSF) but rather the signal level, ${ }^{39,40}$ this is an unexpected property of interferometric spatially incoherent illumination. Therefore, a wavefront sensorless method that relies on the improvement of image quality, which is well adapted to the FFOCT detection, ${ }^{41,42}$ was used for the optimization process. Hence, no wavefront sensor is needed and the setup can be further simplified.

In this paper, we develop and demonstrate a simple, compact, and sensorless AO-FFOCT system for aberrations correction. No well-defined pupil conjugation is needed and a wavefront sensorless algorithm is used with a transmissive LCSLM as aberration corrector. We verified experimentally with an USAF resolution target that system resolution was almost insensitive to aberrations. LCSLM-induced and sample-induced aberration corrections are presented with a negative USAF resolution target as well as biological samples. We finally discuss the potential of this AO-FFOCT for retinal imaging.

\section{Materials and Methods}

\subsection{Adaptive Optics Full-Field Optical Coherence Tomography}

The apparatus schematic diagram is shown in Fig. 1. The main part of the system is the typical FFOCT system based on a Linnik interferometer. An LED with $\lambda=660 \mathrm{~nm}$ center wavelength and 20-nm bandwidth (M660L4, Thorlabs) is used as the incoherent light source. The illumination beam is split into the reference arm and the sample arm at a ratio of 50:50 with a nonpolarizing beamsplitter. Two Nikon 4X/0.2NA Plan APO objectives are used, one is in the sample arm to simulate the open pupil human eye, and the other is in the reference arm. A reference mirror supported by a piezoelectric transducer is placed at the focal plan of the objective in the reference arm while the imaging object is placed in the sample arm. The back-reflected beams from the reference mirror and the sample are recombined by the beamsplitter and imaged with an achromatic doublet lens onto a fast (150 fps) CMOS camera (MV-D1024E-160-CL-12, PhotonFocus). The camera has a resolution of $1024 \times 1024$ pixels with $10.6 \mu \mathrm{m} \times 10.6 \mu \mathrm{m}$ pixel size, and the whole active optical area is used for imaging in experiments demonstrated here. The setup is aligned to ensure that the focusing of the two arms and their optical paths are matched. The piezoelectric transducer creates a four-phase modulation of the reference arm and an FFOCT image can be reconstructed with these four corresponding images. ${ }^{4}$ Usually several FFOCT images are averaged for improving the signal to noise ratio (SNR). Five images were used for the experiments described in this paper requiring about 150-ms acquisition time. The system has a field of view of $1.7 \times 1.7 \mathrm{~mm}^{2}$, and the theoretical resolutions are $2 \mu \mathrm{m}$ (transverse) and $7.7 \mu \mathrm{m}$ (axial). 
For conducting the wavefront correction, a transmissive LCSLM is installed in the sample arm at about $2.5 \mathrm{~cm}$ after the back aperture of the objective lens while another identical LCSLM is set in the reference arm for dispersion correction. A polarizer is inserted in the illumination path since the LCSLM works only with polarized light. By electronically varying the orientation of the molecules inside the pixels of the LCSLM, the refractive index of the pixels is changed independently from each other, resulting in variable retardance abilities to the polarized light passing through them.

\subsection{Resolution Almost Insensitive to Aberrations}

Our recent work on quantifying the effect of geometrical aberrations on incoherent interferometry has shown that the width of the system PSF is almost insensitive to aberrations. ${ }^{39,40}$ In a nutshell, if we consider the aberrated object channel wavefront corresponding to a single point scatter in the "best focus" plane of the sample, because of its partial overlap with the wavefront of the corresponding diffraction limited spot in the reference channel, the signal is damped. Nevertheless, the interferometric signal corresponding to a neighboring diffraction spot is much more damped. Indeed the shift to the neighboring spot in the reference channel is associated to a linear increase of $\pm 2 \pi$ of the phase shift from one side of the pupil to the other. This supplementary phase shift increases the root-mean-square (RMS) wavefront error of the aberrated wavefront leading to a strong decrease of the Strehl ratio and of the signal. Thus, as demonstrated in Ref. [39], the system PSF is computed as a dot product of the object channel PSF with the reference channel PSF for spatially incoherent interferometry while it is computed as a convolution of the object channel PSF with the reference channel PSF for spatially coherent interferometry. So in FFOCT, if the object channel PSF is distorted (mostly broadened), its interference with the reference channel conserves the main feature of an unperturbed PSF with only a reduction in the signal level. Such behavior, which takes advantage of the spatial incoherence of the source, is not likely to happen with scanning OCT setups, which use spatially coherent sources.

With a negative USAF set at the best focus position of the sample arm of our AO-FFOCT system, we confirmed experimentally this specific merit of FFOCT system. Here, a random aberration [Fig. 2(g), Strehl ratio $=0.06$, corresponding to $\mathrm{RMS}=0.27 \lambda]$ was induced with the LCSLM in the sample arm by generating and applying random voltages within the adjusting range across the LCSLM pixels. According to the definition, the "best focus" signal intensity damping compared to the diffraction limited condition is given (for small aberrations) by the Strehl ratio that is proportional to the peak aberrated image intensity. So the Strehl ratio for LCSLM-induced random aberrations/corrections in our experiments with USAF resolution target is actually calculated by $\mathrm{s}=\left(I_{a} / I_{o}\right)^{2}$ as amplitude instead of intensity is obtained for FFOCT signal, where $I_{o}$ is the mean intensity of the FFOCT image before aberration is induced and $I_{a}$ is the mean intensity of aberrated/corrected FFOCT image. Figure 2 shows the sample reflectance images and FFOCT images of the USAF resolution target before and after the random aberration was induced. The reflectance images were recorded by blocking the reference arm in FFOCT; thus, the system works as a wide-field microscope. The reflectance image is blurred after the aberration is added, while there is no obvious blurring of the line patterns in the FFOCT image but only a reduction of the image intensity. The normalized intensity of the selected line in the reflectance image shows a distortion after the aberration was added, while it shows a conservation of the shape for the FFOCT image. Note that the image contrast of scanning OCT using spatially coherent illumination would be close to the reflectance image from the sample arm.

\subsection{Aberration Correction Algorithm}

Since aberrations affect only the signal level without reducing the image resolution in FFOCT, we naturally decide to apply a wavefront sensorless approach for aberration correction based on the FFOCT signal level. The wavefront sensorless method
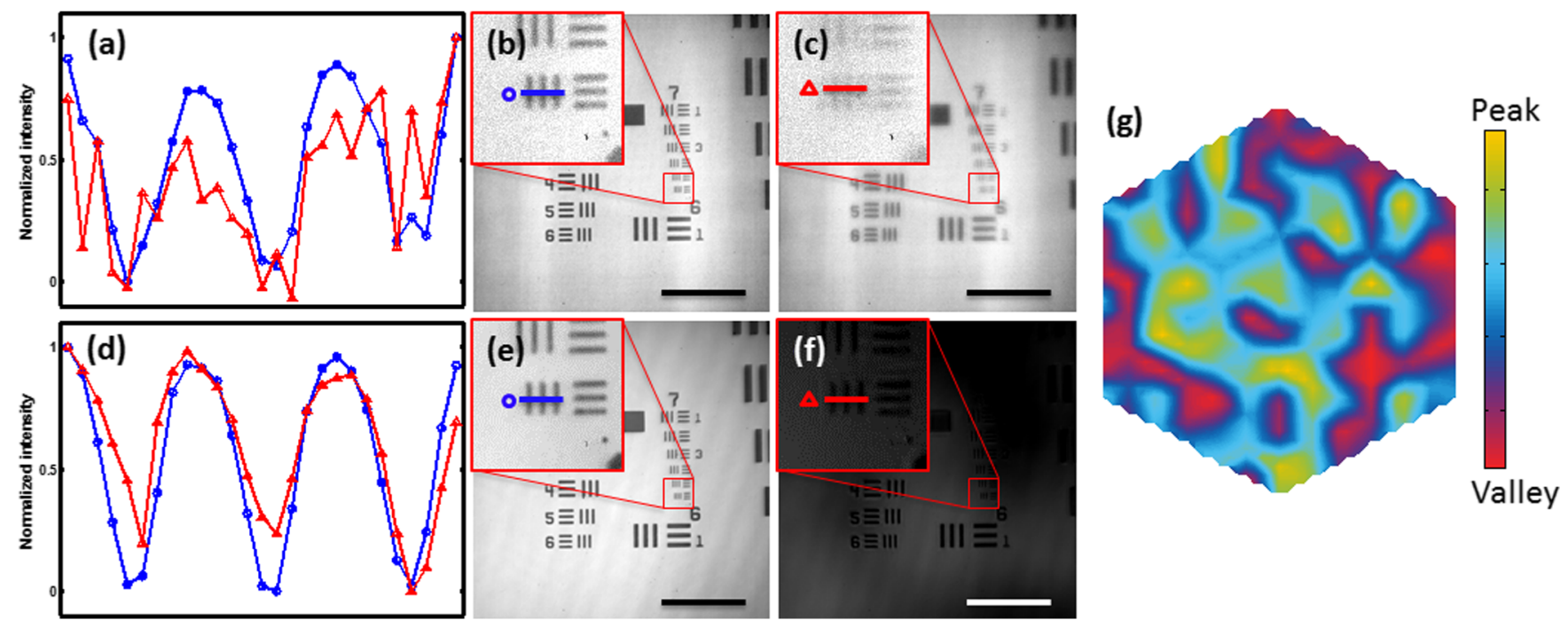

Fig. 2 Comparison of $(b, c)$ the reflectance and (e, f) FFOCT images of a negative USAF resolution target $(b, e)$ before and $(c, f)$ after adding a defocus aberration. (a,d) The comparison of the normalized reflectance intensity and FFOCT signal of the selected line without (blue) and with (red) aberration added. The plot of the random aberration pattern is shown in $(\mathrm{g})$, Strehl ratio $=0.06$. Scale bar: $100 \mu \mathrm{m}$. 


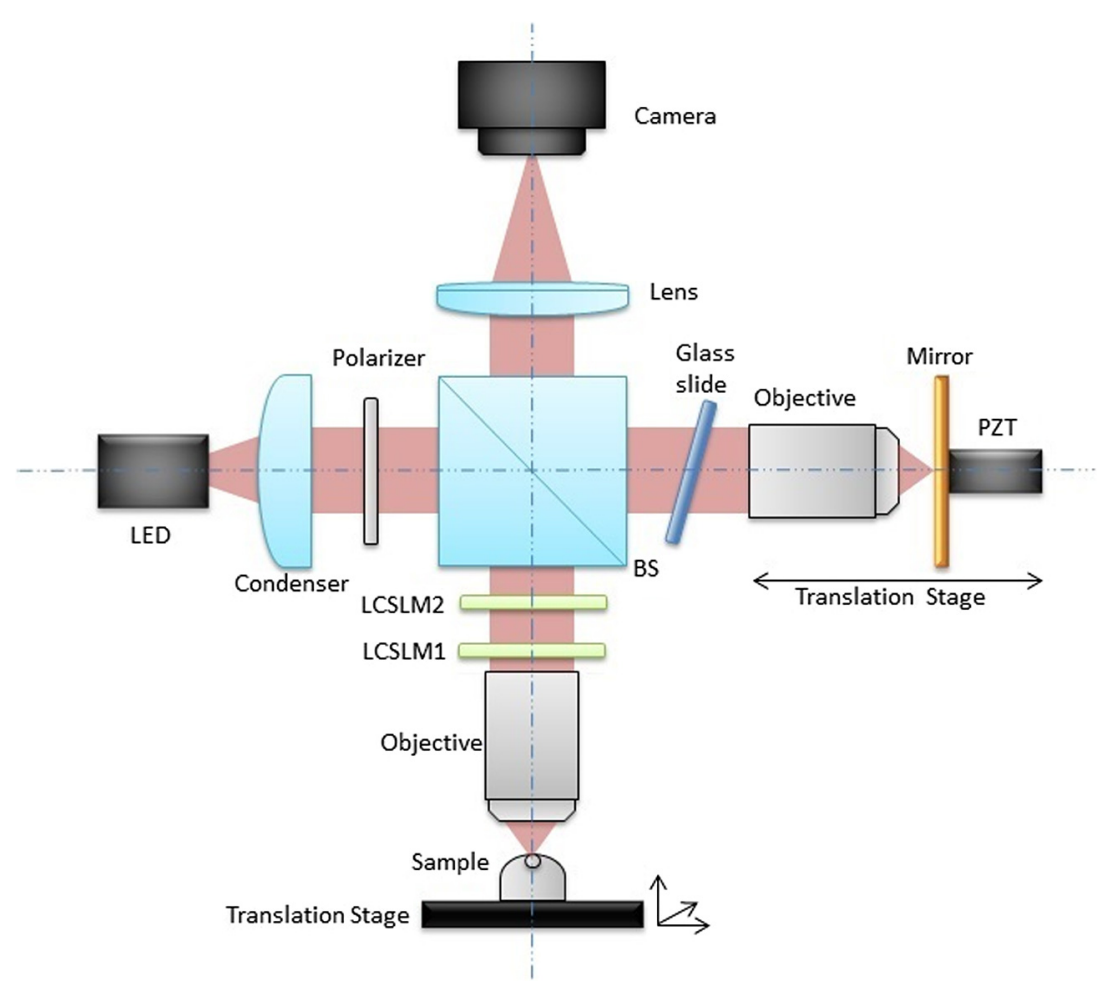

Fig. 3 Schematic of AO FFOCT system for LCSLM-induced aberration correction. LCSLM2 was inserted at $50 \mathrm{~mm}$ after LCSLM1. LCSLM2 was used for aberration introduction while LCSLM1 was used for aberration correction.

consists of the sequential adjustment of the coefficients of loworder orthogonal Zernike polynomial functions applied to the LCSLM to optimize the metric function. In our experiment, the mean intensity of FFOCT image was used as the metric function for LCSLM-induced aberration correction with USAF resolution target as the sample. For in-depth sample-induced aberration correction, the average intensity of the 300 pixels with maximum intensity values in the FFOCT image was used as the metric function because the mean intensity of the overall image would be less sensitive to the AO process since most parts of the FFOCT image has very low or even no signal. Of course we could also restrict to specific region of interest for the optimization process. Indeed anisoplanatism shows up as shown in Fig. 4, but the experiment results we acquired show acceptable correction with this simple AO algorithm. No phase wrapping was used for experiments in this paper because the magnitude of the wavefront distortions to be compensated was within the dynamical range of our SLM. Coefficients were indeed selected within the adjusting range of the LCSLM. The orthogonality of different Zernike modes ensures that the coefficient of each mode for optimal correction is determined independently. ${ }^{43,44}$ This algorithm has been proposed and used by many groups with different wavefront shaping methods and optimization metrics in specific applications. ${ }^{22,45,46}$ For the aberration correction experiments mentioned in this paper, only Zernike modes three to eight were optimized just to demonstrate the feasibility of our system and method. For each mode, FFOCT images were taken for seven different coefficients within the adjusting range. With the extracted metric function values, B-spline interpolations were done and the coefficient that produced the highest metric function was chosen as the correction value. As a result, the entire optimization process could be done in about $6.3 \mathrm{~s}$.

\section{Results}

\subsection{Liquid Crystal Spatial Light Modulator-Induced Aberration Correction}

To test the performances of our AO-FFOCT system with no well-defined conjugation and wavefront sensorless algorithm, experiments of LCSLM-induced aberration correction were first conducted by imaging a negative USAF resolution target. As shown in Fig. 3, in this experiment we inserted LCSLM2 into the sample arm for aberration introduction at about $5 \mathrm{~cm}$ after the original LCSLM1, which was used for aberration correction, thus there is no well-defined conjugation between the aberration introduction plane and the correction plane. A glass slide was inserted into the reference arm for dispersion compensation.

The USAF target was set at the best focus position in the sample arm and a random aberration mask (RMS $=0.23 \lambda$, Strehl ratio $=0.12$ ) was generated and applied to the LCSLM2. Figure 4(a) shows the original FFOCT image with the added aberration. By using the wavefront correction algorithm and applying the correction phase mask onto LCSLM1, defocus, astigmatism, coma, and spherical aberration were corrected sequentially. Figures 4(b)-4(g) show the images after each correction with a clearly visible improvement of image quality after each optimization process. The black curve in Fig. 4(h) shows the increase of the metric function and the red, blue, and green dashed curves display the mean intensity changes of the corresponding selected regions indicated with the same colors in Figs. 4(a) and 4(g). The fact that different levels of improvement were achieved for different regions with the same correction phase mask for each Zernike mode implies the existence of anisoplanatism in our experiment. Nevertheless, the mean intensity of the FFOCT image got an increase of $135 \%$ after the overall 


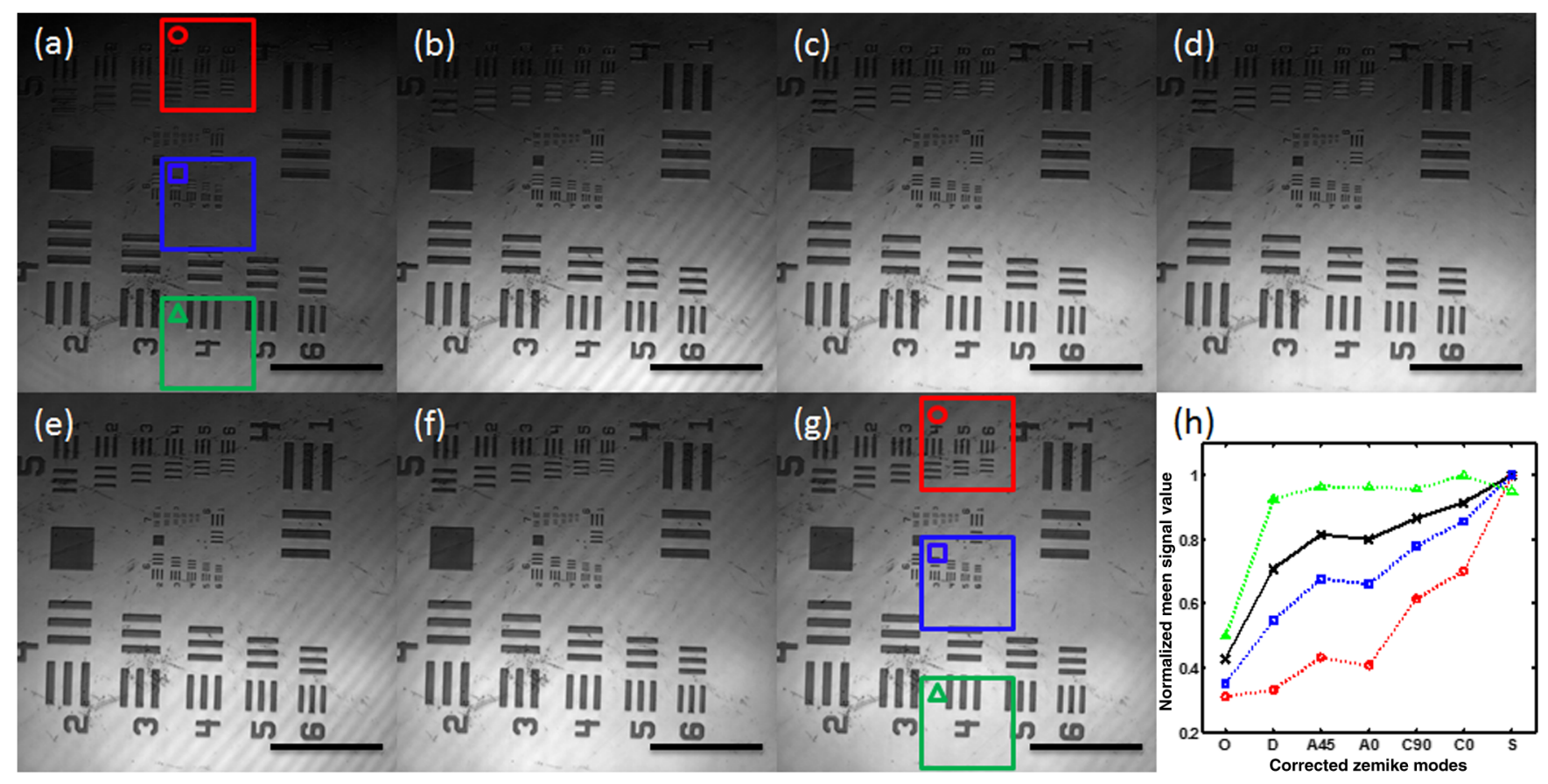

Fig. 4 FFOCT images of a negative USAF resolution target during the nonconjugate AO correction process of a random aberration. (a) Original image with a random aberration added, (b-g) images after defocus, astigmatism 45 , astigmatism 0 , coma 90 , coma 0 , and spherical aberration were corrected, respectively, (h) graph of the metric function (black curve) increase after each correction step and mean intensity changes (red, blue, and green dashed curves) of the corresponding selected regions indicated in $(\mathrm{a}, \mathrm{g})$. Scale bar: $350 \mu \mathrm{m}$.

correction, reaching $80.0 \%$ of the nonaberrated FFOCT image while having diffraction-limited resolution. The RMS wavefront distortion was reduced by a factor of 2.2 to $0.11 \lambda$, and the Strehl ratio was increased by a factor of 5.3 to a value of 0.64 . This experiment was repeated three times in the same condition with different random aberration phase masks added to LCSLM2, and the corrections result in an average increase of the mean intensity to $78.0 \% \pm 2.2 \%$ of the nonaberrated FFOCT image, corresponding to a Strehl of $0.61 \pm 0.035$.

Note that in conjugate $\mathrm{AO}$, the aberration corrector is conjugated with the plane where aberrations dominate. For simplification and avoiding modification of the system, a simulation of conjugate AO experiment by using the same LCSLM for aberration introduction and correction was conducted to correct loworder aberrations for comparison with our nonconjugate $\mathrm{AO}$
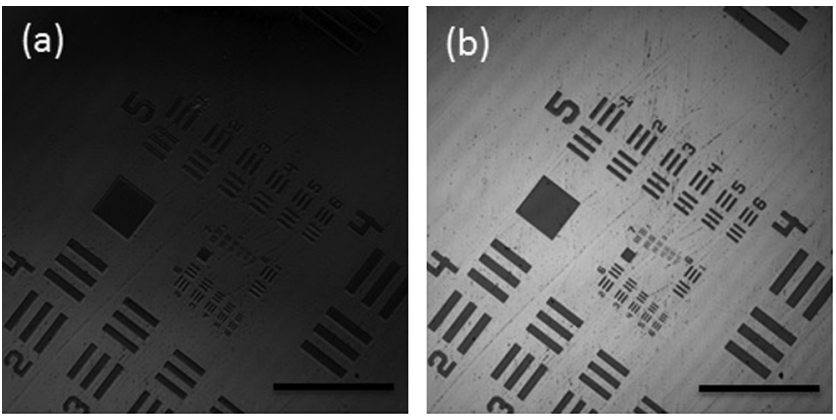

Fig. 5 FFOCT images of a negative USAF resolution target before and after the conjugate $\mathrm{AO}$ correction process of a random aberration. (a) Original image with a random aberration added and (b) image after defocus, astigmatism 45 , astigmatism 0 , coma 90 , coma 0 , and spherical aberration were corrected. Scale bar: $350 \mu \mathrm{m}$. experiments. Again an USAF resolution target was first set at the focal plane in the sample arm. With the same random aberrations induced by LCSLM2 for nonconjugate AO experiments, we demonstrate the aberrations corrections here also on LCSLM2. Indeed the net wavefront of the original aberration phase mask plus the correction phase mask was applied to the LCSLM2 during the correction process. With the same algorithm based on the mean intensity increase, Zernike modes three to eight were blindly corrected. Figure 5 shows the correction of the same random aberration corresponding to the results displayed in Fig. 4, the whole correction result in the mean intensity of the FFOCT image reaching $86.0 \%$ of the nonaberrated FFOCT image. The RMS wavefront distortion was reduced by a factor of 2.6 to $0.09 \lambda$, and the Strehl ratio was increased by a factor of 6.2 to a value of 0.74 . The three repeated experiments result in an average increase of the mean intensity to $84.3 \% \pm 2.1 \%$ of the nonaberrated FFOCT image, corresponding to a Strehl of $0.71 \pm 0.036$.

\subsection{Sample-Induced Aberrations Correction}

Due to the spatial variations of refractive index within biological samples and surface topography, aberration distortion is severe when imaging into the sample volume. In order to further demonstrate the feasibility of our system and method even for weak aberration correction, experiments of sample-induced aberration corrections were done with a ficus leaf. The system setup described in Fig. 1 was used here. By imaging at a depth of $75 \mu \mathrm{m}$ under the leaf surface, only weak aberrations are induced and we can, thus, check the sensitivity of our correction approach; the low-order contents of the self-induced sample aberrations were corrected step-by-step with the aforementioned 
(a)
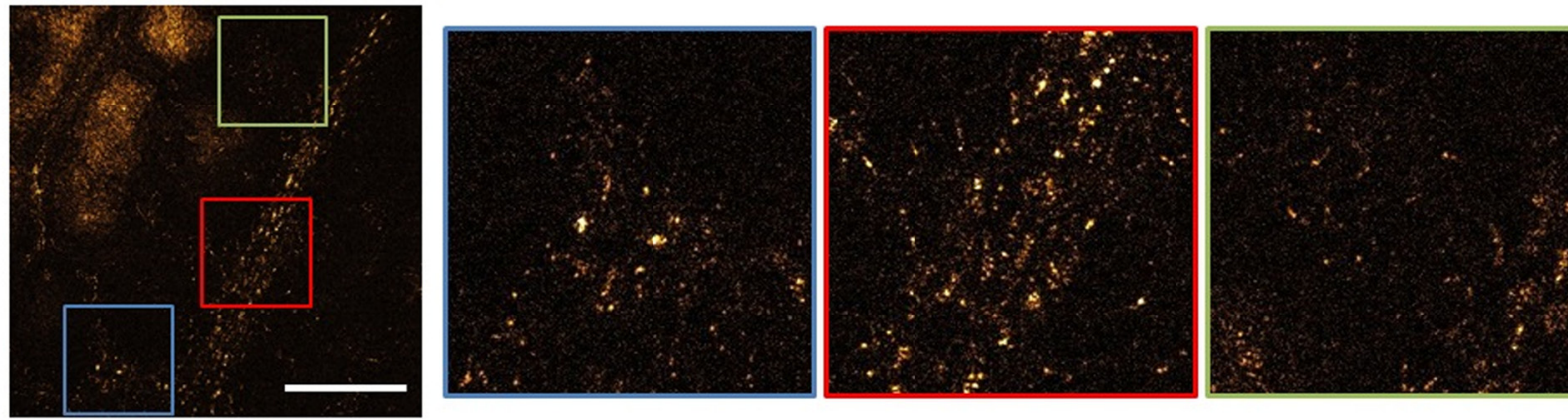

High

(b)
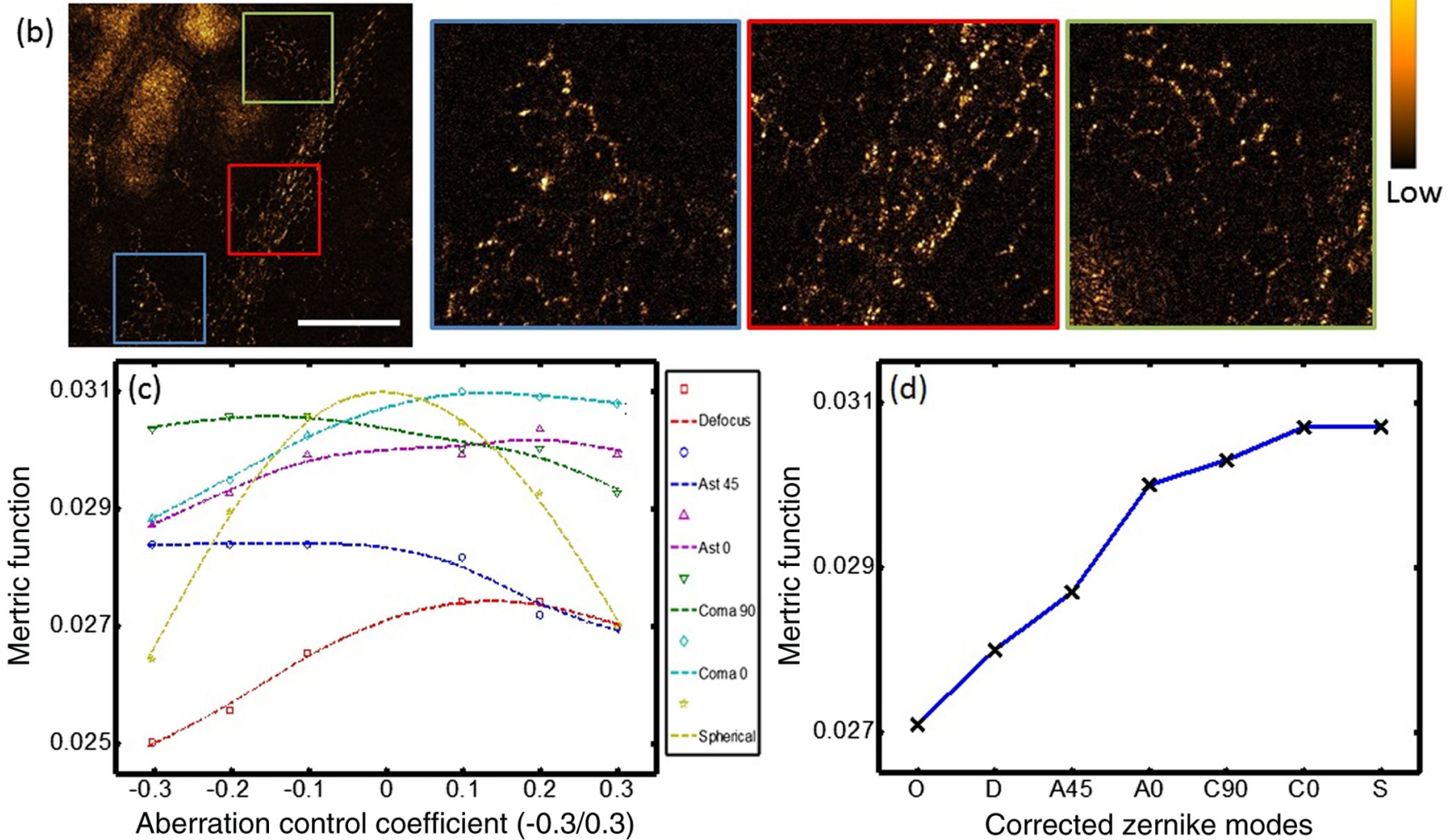

Fig. 6 Comparison of FFOCT images of a ficus leaf (a) before and (b) after sample self-induced aberration was corrected when imaging at a depth of $75 \mu \mathrm{m}$. (c) Graph of the metric function during the optimization process and (d) graph of the metric function increase after each correction step. Scale bar: $500 \mu \mathrm{m}$, Zoomed in area: $425 \times 425 \mu \mathrm{m}$.

methods. As showed in Fig. 6, the optimized image [Fig. 6(b)] shows an intensity increase compared with the original image [Fig. 6(a)] and from the zoomed in images, more structured information appears. This is due to the fact that the correction process increased the SNR and more signals that were buried by the noise before appear after the AO correction. The graph of the metric function while adjusting the coefficients of each Zernike mode is displayed in Fig. 6(c). The highest positions of each curve correspond to the coefficients used for the optimal correction of each mode. Figure 6(d) shows the increase of metric function. The whole correction process results in $13.3 \%$ improvement of the metric function. As expected the metric function improvement increases to $35.5 \%$ when imaging deeper at $120 \mu \mathrm{m}$ under the leaf surface in another experiment (not shown here).

After showing the ability of our approach to optimize the signal even with a low level of aberration, we checked another biological tissue of relevance that suffers from strong scattering and stronger aberrations is brain tissue, where FFOCT signal is usually strongly reduced when imaging deep in the sample. We have also conducted experiments with a fixed mouse brain tissue slice to correct the wavefront distortion. Imaging was performed at $50 \mu \mathrm{m}$ under the brain tissue surface without liquid matching fluid, and the results are shown in Fig. 7. The high-signal
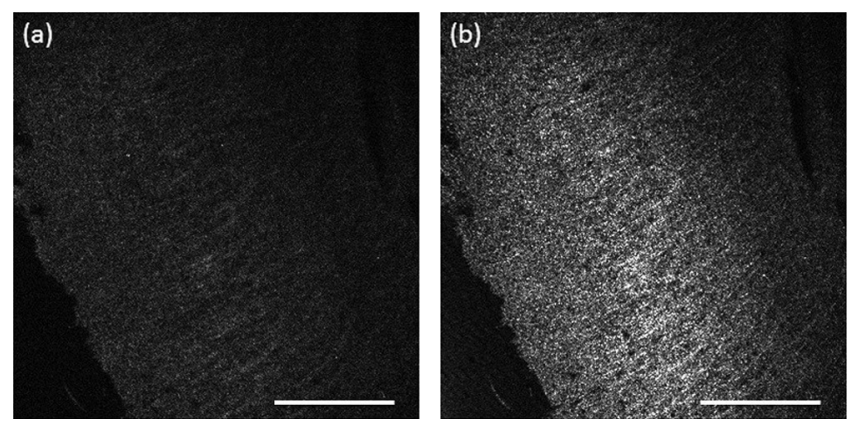

Fig. 7 Comparison of FFOCT images of fixed mouse brain tissue slice (a) before and (b) after sample self-induced aberration was corrected when imaging at a depth of $50 \mu \mathrm{m}$. Scale bar: $500 \mu \mathrm{m}$. 
fiber-like myelin fiber structures appeared much more clearly after the whole correction process because of the increased SNR; indeed the metric function was increased by $121 \%$.

\section{Discussion and Conclusion}

Here, we demonstrated that a compact transmissive LCSLM can be directly coupled to an FFOCT system as an AO element for wavefront distortion compensation with a wavefront sensorless algorithm. Our experiments show the potential of this compact AO-FFOCT system for aberration correction imaging.

The conjugation of the LCSLM with the pupil plane was discarded in our AO-FFOCT system. Traditionally, AO devices are usually conjugated with a well-defined plane. For both pupil $\mathrm{AO}$, in which conjugation is done to the pupil plane, and conjugate $\mathrm{AO}$, in which conjugation is done to the plane where the aberrations dominate, a plane is needed for wavefront measurement and the inverse phase mask needs to be applied to the same plane with the conjugated wavefront correctors. The advantages and disadvantages of both conjugations have been recently discussed. ${ }^{47}$ From what we have learned in our experiments, we think that the problem might be easier for applications with metric-based wavefront sensorless $\mathrm{AO}$ because the only criteria are the metric functions of the image. Strict conjugation might be able to be abandoned, especially for low-order aberration correction cases. The corrected signal level with this nonconjugate AO reaches $78.0 \% \pm 2.2 \%$ of the nonaberrated situation. This is slightly inferior but still acceptable compared with a conjugate AO experiment, which results in a corrected FFOCT image signal level reaching $84.3 \% \pm 2.1 \%$ of the nonaberrated image.

With spatially incoherent illumination, we qualitatively demonstrated that FFOCT resolution is almost insensitive to aberrations with only signal reduction due to the limitation of the camera pixels and system magnification. But this specific merit of FFOCT is quantitatively discussed in Ref. 39. More precisely the aberration-induced reduction in FFOCT signal is roughly proportional to the square root of the Strehl ratio as amplitude is taking as the FFOCT signal. Our approach simulating eye aberrations correction in a simple manner opens the path to a straightforward implementation of AO-FFOCT for retinal examinations in our future research. In eye examination, we think that we can restrict aberrations correction to main aberrations (e.g., focus and astigmatism) that will improve the SNR and skip the high-order aberrations. Ultimately, the lens in the eyeball will play the role of the objective used in the sample arm in our experiments; therefore, a new reference arm with path and dispersion compensation ${ }^{48}$ will need to be designed taking into consideration of the eye characteristics.

\section{Acknowledgments}

We thank Ignacio Izeddin for fruitful comments and discussions. This work was supported by the HELMHOLTZ Synergy funded by the European Research Council (ERC).

\section{References}

1. T. Wilson, Confocal Microscopy, Vol. 426, pp. 1-64, Academic Press, London (1990).

2. W. Denk, J. H. Strickler, and W. W. Webb, "Two-photon laser scanning fluorescence microscopy," Science 248(4951), 73-76 (1990).

3. D. Huang et al., "Optical coherence tomography," Science 254(5035), 1178-1181 (1991).

4. L. Vabre, A. Dubois, and A. C. Boccara, "Thermal-light full-field optical coherence tomography," Opt. Lett. 27(7), 530-533 (2002).
5. H. Babcock, "The possibility of compensating atmospheric seeing," Publ. Astron. Soc. Pac. 65, 229-236 (2010).

6. G. Rousset et al., "First diffraction-limited astronomical images with adaptive optics," Astron. Astrophys. 230, L29-L32 (1990).

7. J. A. Kubby, Adaptive Optics for Biological Imaging, CRC Press, Boca Raton, Florida (2013).

8. P. Godara et al., "Adaptive optics retinal imaging: emerging clinical applications," Optom. Vision Sci. 87(12), 930-941 (2010).

9. J. Porter et al., Eds., Adaptive Optics for Vision Science, John Wiley \& Sons, Inc., Hoboken, New Jersey (2006).

10. D. R. Williams, "Imaging single cells in the living retina," Vision Res. 51(13), 1379-1396 (2011).

11. J. Liang, D. R. Williams, and D. T. Miller, "Supernormal vision and high-resolution retinal imaging through adaptive optics," J. Opt. Soc. Am. A 14(11), 2884-2892 (1997).

12. A. Roorda et al., "Adaptive optics scanning laser ophthalmoscopy," Opt. Express 10(9), 405-412 (2002).

13. Y. Zhang and A Roorda, "Evaluating the lateral resolution of the adaptive optics scanning laser ophthalmoscope," J. Biomed. Opt. 11(1), 014002 (2006).

14. D. Merino et al., "Observation of cone and rod photoreceptors in normal subjects and patients using a new generation adaptive optics scanning laser ophthalmoscope," Biomed. Opt. Express 2(8), 2189-2201 (2011).

15. Y. Zhang et al., "High-speed volumetric imaging of cone photoreceptors with adaptive optics spectral-domain optical coherence tomography," Opt. Express 14(10), 4380-4394 (2006).

16. R. J. Zawadzki et al., "Adaptive-optics optical coherence tomography for high-resolution and high-speed 3D retinal in vivo imaging," Opt. Express 13(21), 8532-8546 (2005).

17. O. P. Kocaoglu et al., "Imaging cone photoreceptors in three dimensions and in time using ultrahigh resolution optical coherence tomography with adaptive optics," Biomed. Opt. Express 2(4), 748-763 (2011).

18. Y. Zhang et al., "Adaptive optics parallel spectral domain optical coherence tomography for imaging the living retina," Opt. Express $\mathbf{1 3}(12)$, 4792-4811 (2005).

19. E. J. Fernández et al., "Ultrahigh resolution optical coherence tomography and pancorrection for cellular imaging of the living human retina," Opt. Express 16, 11083-11094 (2008).

20. E. J. Fernández et al., "Three-dimensional AO ultrahigh-resolution optical coherence tomography using a liquid crystal spatial light modulator," Vision Res. 45, 3432-3444 (2005).

21. O. P. Kocaoglu et al., "Adaptive optics optical coherence tomography with dynamic retinal tracking," Biomed. Opt. Express 5(7), 2262-2284 (2014).

22. K. S. K. Wong et al., "In vivo imaging of human photoreceptor mosaic with wavefront sensorless adaptive optics optical coherence tomography," Biomed. Opt. Express 6(2), 580-590 (2015).

23. M. Rueckel, J. A. Mack-Bucher, and W. Denk, "Adaptive wavefront correction in two-photon microscopy using coherence-gated wavefront sensing," Proc. Natl. Acad. Sci. U. S. A. 103(46), 17137-17142 (2006).

24. S. H. Lee, J. S. Werner, and R. J. Zawadzki, "Improved visualization of outer retinal morphology with aberration cancelling reflective optical design for adaptive optics - optical coherence tomography," Biomed. Opt. Express 4(11), 2508-2517 (2013).

25. J. Porter et al., "Monochromatic aberrations of the human eye in a large population," J. Opt. Soc. Am. A 18(8), 1793-1803 (2001).

26. J. F. Castejón-Mochón et al., "Ocular wave-front aberration statistics in a normal young population," Vision Res 42(13), 1611-1617 (2002).

27. X. Hong et al., "Statistics of aberrations among healthy young eyes," in Vision Science and Its Applications, OSA Trends in Optics and Photonics, Optical Society of America, paper SuA5 (2001).

28. D3128 Spatial Light Modulator, Meadowlark Optics http://www. meadowlark.com/.

29. G. D. Love, "Wave-front correction and production of Zernike modes with a liquid-crystal spatial light modulator," Appl. Opt. 36(7), 15171524 (1997).

30. A. Vyas et al., "Spatial light modulator for wavefront correction," arXiv preprint arXiv: 0909.3413 (2009).

31. L. N. Thibos and A. Bradley, "Use of liquid-crystal adaptive-optics to alter the refractive state of the eye," Optom. Vision Sci. 74(7), 581-587 (1997).

32. F. Vargas-Martın et al., "Correction of the aberrations in the human eye with a liquid-crystal spatial light modulator: limits to performance," J. Opt. Soc. Am. A 15(9), 2552-2562 (1998). 
33. N. Doble et al., "Requirements for discrete actuator and segmented wavefront correctors for aberration compensation in two large populations of human eyes," Appl. Opt. 46(20), 4501-4514 (2007).

34. D. T. Miller, L. N. Thibos, and X. Hong, "Requirements for segmented correctors for diffraction-limited performance in the human eye," Opt. Express 13(1), 275-289 (2005).

35. P. N. Marsh, D. Burns, and J. M. Girkin, "Practical implementation of adaptive optics in multiphoton microscopy," Opt. Express 11(10), 1123-1130 (2003).

36. L. Sherman et al., "Adaptive correction of depth-induced aberrations in multiphoton scanning microscopy using a deformable mirror," J. Microsc. 206, 65-71 (2002).

37. S. Zommer et al., "Simulated annealing in ocular adaptive optics," Opt. Lett. 31(7), 939-941 (2006).

38. N. Ji, D. E. Milkie, and E. Betzig, "Adaptive optics via pupil segmentation for high-resolution imaging in biological tissues," Nat. Methods 7(2), 141-147 (2009).

39. P. Xiao, M. Fink, and A. C. Boccara, "Full-field spatially incoherent interferometry: a spatial resolution almost insensitive to aberrations," Opt. Lett. 41(17), 3920-3923 (2016).

40. P. Xiao, M. Fink, and A. C. Boccara, "An optical tomography PSF almost insensitive to aberrations: the benefit of a spatial incoherent illumination (conference presentation)," Proc. SPIE 9717, 97170I (2016).

41. A. Dubois, G. Moneron, and A. C. Boccara, "Thermal-light full-field optical coherence tomography in the $1.2 \mu \mathrm{m}$ wavelength region," Opt. Commun. 266(2), 738-743 (2006).

42. S. Labiau et al., "Defocus test and defocus correction in full-field optical coherence tomography," Opt. Lett. 34(10), 1576-1578 (2009).

43. R. J. Noll, "Zernike polynomials and atmospheric turbulence," J. Opt. Soc. Am. 66(3), 207-211 (1976).

44. G. Dai, Wavefront Optics for Vision Correction, SPIE Press, Bellingham, Washington (2008).

45. S. Bonora and R. J. Zawadzki, "Wavefront sensorless modal deformable mirror correction in adaptive optics: optical coherence tomography," Opt. Lett. 38(22), 4801-4804 (2013).
46. D. Debarre, M. J. Booth, and T. Wilson, "Image based adaptive optics through optimization of low spatial frequencies," Opt. Express 15(13), 8176-8190 (2007).

47. J. Mertz, H. Paudel, and T. G. Bifano, "Field of view advantage of conjugate adaptive optics in microscopy applications," Appl. Opt. 54(11), 3498-3506 (2015).

48. C. K. Hitzenberger et al., "Dispersion effects in partial coherence interferometry: implications for intraocular ranging," J. Biomed. Opt. 4(1), 144-151 (1999).

Peng Xiao is currently working at the Institut Langevin (Paris, France) as a PhD student. He received his BEng degree from Harbin Institute of Technology (Harbin, China) and his MSc degree from Pohang University of Science and Technology (Pohang, South Korea). His current research interests include full-field OCT (FFOCT), with a focus on adaptive optics FFOCT for high-resolution retinal imaging.

Mathias Fink received a PhD in solid-state physics in 1970 and received the doctorates-sciences degree in 1978 from Paris University. His current research interests include medical ultrasonic imaging, ultrasonic therapy, nondestructive testing, underwater acoustics, telecommunications, seismology, active control of sound and vibration, analogies between optics, quantum mechanics, and acoustics, wave coherence in multiply scattering media, and time-reversal in physics. He holds 28 patents and has published more than 300 articles.

Albert Claude Boccara was dean of research at ESPCI-ParisTech. Among the optical methods he has developed new microscopies to increase depth and lateral resolution. Recently, ultimate measurements have found new fields of application going from detection of gravitational waves to $3-\mathrm{D}$ imaging though scattering media. He has published more than 300 scientific articles (ISI/ Boccara $A^{*}$ or Boccara ()) in international journals. In 2007 he founded the startup LLTech devoted to medical imaging and diagnostics. 